SMCia/99

1999 IEEE Midnight-Sun Workshop on Soft Computing Methods in Industrial Applications

Kuusamo, Finland, June 16-18, 1999

\title{
Qualitative phase space modeling of non-linear electrical dynamic systems
}

\author{
W.K. Tang \\ Department of Electrical Engineering \\ The Hong Kong Polytechnic University \\ Hung Hom, Kowloon, Hong Kong \\ eewktang@inet.polyu.edu.hk
}

\author{
Y.K. Wong \\ Department of Electrical Engineering \\ The Hong Kong Polytechnic University \\ Hung Hom, Kowloon, Hong Kong \\ eeykwong(a)polyu.edu.hk
}

\author{
A.B. $\operatorname{Rad}$ \\ Department of Electrical Engineering \\ The Hong Kong Polytechnic University \\ Hung Hom, Kowloon, Hong Kong \\ eeabrad@polvu.edu.hk
}

\begin{abstract}
The purpose of this paper is to combine the existing methodologies that describe non-linear dynamic systems, by using the phase space and the descriptions of qualitative behaviors of ordinary differential equations. The swing equation of the synchronous machine has been illustrated as an example.
\end{abstract}

\section{INTRODUCTION}

Most dynamic systems are non-linear in the nature, and some cannot be solved by analytical methods and they will not obey the superposition principle [10]. Traditional methods are using the linear approximations to represent the systems, but they can only describe a small region of the non-linear systems. Then, it is important to consider what qualitative information can be obtained about these systems without actually solving the equations. In this paper, the qualitative behaviors of a non-linear dynamic system will be interpreted to describe the system behaviors automatically. One approach is that the set of all possible behaviors of the systems is represented by the phase portrait.

Sometimes, it is due to the lack of sufficient quantitative information, so the traditional quantitative techniques for analyzing or modeling are impossible. However, the qualitative analysis can still provide results for the possible outcomes of the given qualitative information. For the current technology, engineering design process is using complex computational tools [3] and computers to numerically analyze and model the systems. Nevertheless, we still need to have the qualitative understanding of the phenomena of the systems to verify those outcomes whether they make sense. Therefore, we need a new method to help us to analyze and model nonlinear systems in a qualitative way.

The methodologies can be applied to some famous nonlinear systems, such as Van der Pol oscillator, the effect of Coulomb friction in the step response of position control and the swing equations for a synchronous machine. The simplified swing equation, which is a second order nonlinear dynamic system, has been selected for case study in this paper.

The existing considerations on the case studies are divided into only a subset of the overall system behaviors. If there are different operating regions, the system dynamics can have different behaviors. This situation is similar to the tale of blinds to model an elephant. To consider the different operating regions, we can use the phase space method to specify the interested operating region, which can be partitioned into several sub-regions where the trajectories have common boundary conditions. The phase portrait of swing equation can be plotted by transforming the equation into two first order differential equations in Cartesian form. Then, we can interpret and analyze the behaviors of the system by the parameters in the equations. The qualitative phase portraits by two different approaches have been applied to the swing equation in this paper.

\section{THEORY}

\section{A. Qualitative Reasoning}

Qualitative reasoning is a method to describe the physical world by incomplete knowledge [2]. All the variables in the qualitative reasoning are expressed in both magnitudes and directions. Most of the "expert knowledge" and "commonsense" are not complete and able to be expressed in equations. Without the exact quantitative information and known relationship between system variables of the physical system, the qualitative reasoning can still describe all the possible phenomena by the qualitative variables. The framework of the relationship between differential equations and qualitative representations is shown in Fig. 1 [2].

\section{B. The QSIM Representation}

The parameters of a model are continuous differentiable functions in time domain. Each of them uses an ordered set of landmark values to illustrate the quantity spaces in discrete regions, such that the parameters have the same 


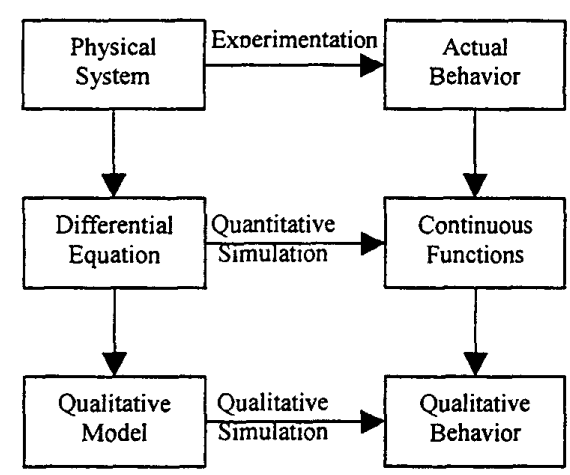

Fig. 1: Framework of qualitative reasoning

characteristics within that region. Landmark values can also be in symbolic form and show there are critical values in itself or in other parameters. The directions of the parameters are part of the qualitative variables. Models can be in different operating regions, which can have their own constraints and qualitative variables. The conditions for the qualitative variables should be defined so that there are transitions from one operating region to another operating region.

$$
Q V\left(f_{i}, t_{i}\right)=<q m a g, q d i r>
$$

where

qmag The magnitude of the qualitative variable, and it can be either the landmark value (point) or between two adjacent landmark values (an interval). For example, the set of landmarks can be $\{-\infty, 0,+\infty\}$.

qdir It represents the direction of the variable as follows:

$$
\text { qdir }= \begin{cases}\text { inc } & \text { if } f_{i}^{\prime}\left(t_{i}\right)>0 \\ \text { std } & \text { if } f_{i}^{\prime}\left(t_{i}\right)=0 \\ \text { dec } & \text { if } f_{i}^{\prime}\left(t_{i}\right)<0\end{cases}
$$

QSIM algorithm [2] reasons those physical systems as autonomous by the qualitative differential equations (QDE). QSIM uses well-defined qualitative constraints to describe the system of QDEs, which predicts the possible behaviors of a system. In the QSIM, the ordered landmark values can be asserted for different variables and can be created during the process while there is a new meaningful critical value found in the interval.

Non-intersection-of-phase-space-trajectory constraint (non-intersection constraint) [11] is a filter of QSIM.
There are still some useful constraints have been employed, such as curvature constraints and higher-order derivative constraint. It embodies the requirements of phase space analysis into the QSIM predictions to reduce the number of spurious behaviors.

\section{Phase Space Analysis}

Phase space analysis is a traditional method to illustrate the behavior of a second order differential equation. It is the Cartesian plot for the variable versus its derivative. The trajectories vary with time from the starting points, which is dependent on the initial conditions. For those differential equations that cannot be solved analytically, the behaviors can still be illustrated in the phase portrait. The phase space analysis shows the behaviors of the system without really solving the differential equations, so it has been widely used in those systems that contain non-linear differential equations. Usually, the analysis is applied to an autonomous system. An autonomous system is independent of time while the input is fixed as constant. The general form of state-space models [6] is described as (1). $\mathrm{X}$ is the set of state variable of the system, $u$ is the inputs from any external systems and $Y$ is the set of output variables from the system. Except for critical points, all trajectories do not cross itself [5],[11].

A second order differential equation is able to be transformed to a set of first order differential equations and be an autonomous system.

$$
\begin{aligned}
\frac{d X}{d t} & =A X+B u \\
Y & =C X+D u
\end{aligned}
$$

There exist some common characteristics in the phase space analysis that help us to understand the behaviors. The behaviors near the fixed points can be determined by the Jacobian matrix [5]. Fixed points (singular points) can be found. According to the eigenvalues and eigenvectors at the fixed points, the types of fixed points can be sorted into stable, half stable or unstable node. Specifically, the fixed points can be classified into centers (of limit cycles), saddle points, and spiral points. After understanding the types of fixed points, we know how the system behaved near the fixed points in time domain. For example, the spiral and center points are stable nodes in the Fig. 2a and Fig. 2c, and the node (Fig. 2d) is unstable when it is diverging in time domain. To draw the phase portrait, the method of isoclines is useful to sketch a trajectory. All trajectories pass through the same isoclines have the same slope. 


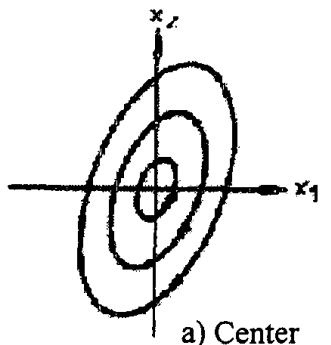

a) Center

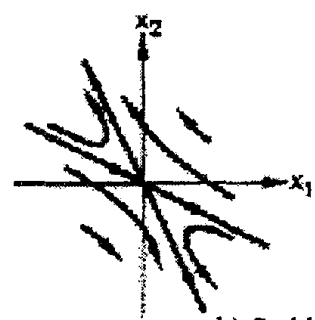

b) Saddle

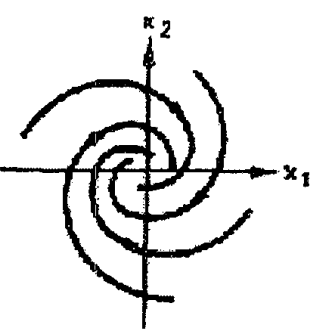

c) Spiral

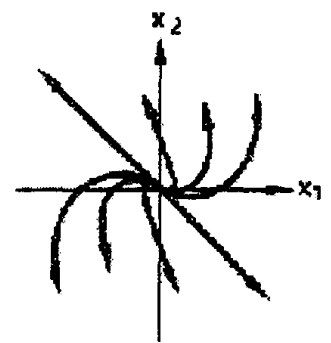

d) Unstable node
Fig. 2: The common fixed points

\section{Swing Equation}

The stability analysis of synchronous machines is very important in electrical engineering. There are some ways to analyze the stability of the synchronous machines. Most of them consider the relationship between the power and the rotor angle, for examples, the equal-area criterion and the swing equation. Transient analysis, which is for the electrical fault, can help engineers to determine the steady-state condition for stability limit.

Swing equation is the description of a synchronous machine, which is driven by a mechanical system and generating power to an infinite busbar. Equation (2) is the simplified swing equation that without friction, magnetic saturation, and variation of field strength and saliency.

$$
H \ddot{\delta}=P_{m}-P_{e} \sin \delta
$$

where $\delta$ is the rotor angle, $H$ is the inertia constant, $P_{m}$ is the mechanical power supplied, and $\mathrm{P}_{\mathrm{e}}$ denotes the maximum electrical power, which can be generated. Obviously, it is a second order nonlinear dynamic system. The phase portrait of swing equation can be plotted by transforming the equation into two first order differential equations in Cartesian form [3]. Then, we can interpret and analyze the behaviors of the system by the parameters in the equations. For examples, after the analysis, we can determine the machine is a generator or motor and where is the equilibrium.

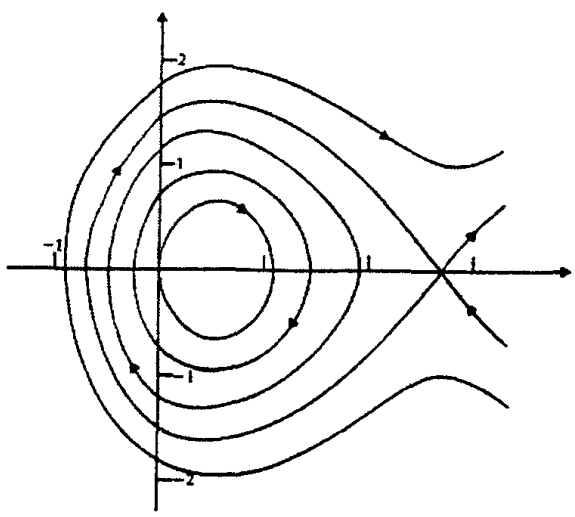

Fig. 3: The numerical simulation of Swing Equation $(\mathrm{H}=1$, $P_{m}=1$ and $P_{c}=2$ )

\section{NUMERICAL METHODS}

As the power of computer increasing, solving non-linear differential equations by numerical methods is very common. Trajectories can be plotted in the phase portrait by the traditional numerical methods. The initial states, however, have to be stated before simulations. The analysis has to be done before making any initial states or they can be chosen randomly. Therefore, it is possible that some of the behaviors would be missed.

The first order system differential equation (3) is rewritten from (2). The variable $x_{1}$ is $\delta$ and $x_{2}$ is derivative of $\delta$. Those variables other than $x_{1}$ and $x_{2}$ are asserted numerical values. If analyses have been done before making any initial states, the number of try and errors can be reduced and the chosen initial points produce some trajectories that represent the critical behaviors of the model. The Fig. 3 is a phase portrait by numerical simulations in several initial states. The arrows indicate the direction of the trajectories with time changing.

$$
\begin{aligned}
& \dot{x}_{1}=x_{2} \\
& \dot{x}_{2}=\frac{P_{m}}{H}-\frac{P_{e}}{H} \operatorname{Sin}\left(x_{1}\right)
\end{aligned}
$$

\section{Qualitative Methods}

In this session, the two kinds of qualitative analysis related to phase space would be applied in the simplified swing equation. The first one is qualitative phase portrait [7] and the second one is qualitative simulation [2],[11]. After the qualitative phase portrait finished, the QSIM simulates the model in time domain with some initial states and draw the corresponding phase spaces, then we can examine the model. 


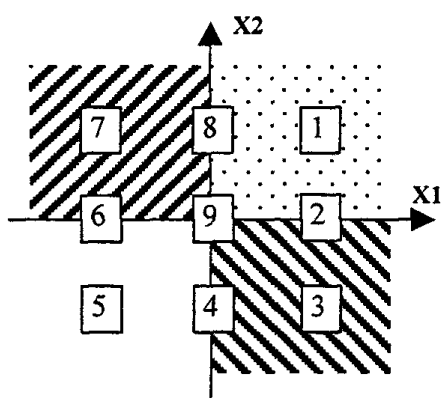

Fig. 4: The Phase Plane Regions

\section{A. Qualitative Phase Portrait}

The qualitative phase portrait is illustrated directly in the phase space without the time domain analysis. The relationship of variables would be examined such that all the possible behaviors are found from the qualitative confluence[4]. The consideration of the confluence applied to partition the qualitative phase space into subregions, where the directions of qualitative variables would be same in the sub-regions.

In the simple qualitative phase space Fig.4, there are two axes that partition the whole space into four regions already. The quantity spaces of $\left[\mathrm{X}_{1}\right]$ and $\left[\mathrm{X}_{2}\right]$ are in the set $\{[-],[0],[+]\}$. Therefore, nine $(3 \times 3)$ basic states locate in the combination of the two variables, $\left[\mathrm{X}_{1}\right]$ and $\left[\mathrm{X}_{2}\right]$ in each phase portrait.

The set of confluence (4) is rewritten from (3) and then one set of the direction tables can be found and shown in TABLE 1 and TABLE 2.

$$
\begin{aligned}
& \partial \mathrm{X}_{1}=\left[\mathrm{X}_{2}\right] \\
& \partial \mathrm{X}_{2}=[\mathrm{A}]-[\mathrm{B}]\left[\operatorname{Sin}\left(\mathrm{X}_{1}\right)\right]
\end{aligned}
$$

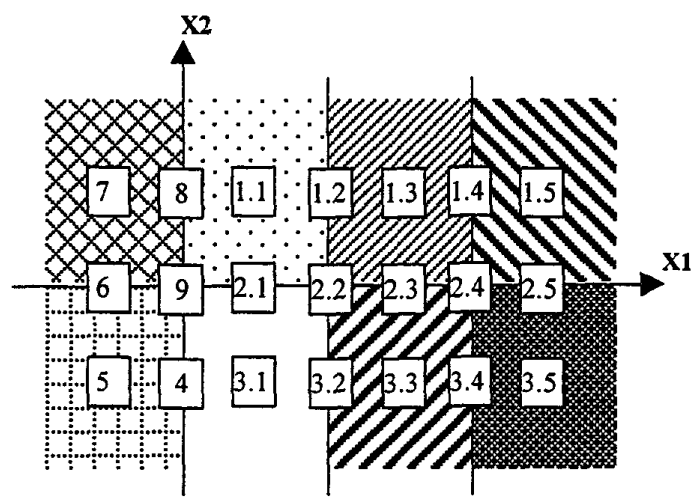

Fig. 5: The Phase Plane Regions of Simplified Swing Equation with state numbers

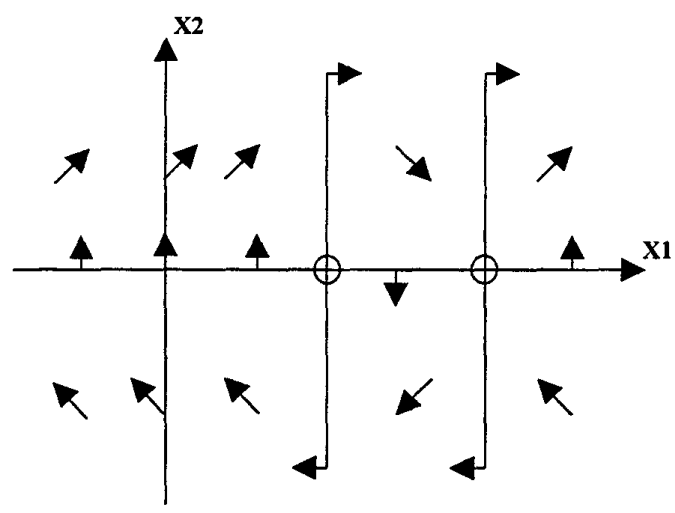

Fig. 6: The qualitative phase portrait

TABLE 1: DIRECTION TABLE OF THE HORIZONTAL COMPONENTS

\begin{tabular}{|c|c|c|c|}
\cline { 2 - 4 } \multicolumn{1}{c|}{} & \multicolumn{3}{|c|}{$\mathrm{X}_{2}$} \\
\cline { 2 - 4 } & $<0$ & 0 & $>0$ \\
\hline$\partial \mathrm{X}_{1}$ & - & 0 & + \\
\hline
\end{tabular}

TABLE 2: DIRECTION TABLE OF THE VERTICAL COMPONENTS

\begin{tabular}{|c|c|c|c|c|c|c|c|}
\cline { 2 - 8 } \multicolumn{1}{c|}{} & \multicolumn{7}{c|}{$\mathrm{X}_{1}$} \\
\cline { 2 - 8 } & $<0$ & 0 & $0<\mathrm{X}_{1}<c_{1}$ & $\mathrm{c}_{1}$ & $\mathrm{c}_{1}<\mathrm{X}_{1}<\mathrm{c}_{2}$ & $\mathrm{c}_{2}$ & $>\mathrm{c}_{2}$ \\
\hline$\partial \mathrm{X}_{2}$ & + & + & + & 0 & - & 0 & + \\
\hline
\end{tabular}

TABLE 3: THE DEFINITION OF [SIN $(X)]$

\begin{tabular}{|c|c|c|c|c|c|}
\hline \multicolumn{1}{|c|}{ TABLE 3: THE DEFINITION OF [SIN $(X)]$} \\
\hline $\begin{array}{c}\text { Type of } \\
\text { Constraints }\end{array}$ & $\begin{array}{c}\text { Landmark } \\
\text { values of X }\end{array}$ & $\begin{array}{c}\text { Landmark } \\
\text { values of } \\
{[\operatorname{SIN}(X)]}\end{array}$ & \multicolumn{3}{|c|}{$\begin{array}{c}\text { Corresponding value pairs } \\
(X, \operatorname{SIN}(X))\end{array}$} \\
\hline$U \cdot$ & $\{0, \pi / 2, \pi\}$ & $\{0,1\}$ & $(0,0)$ & $(\pi / 2,1)$ & $(\pi, 0)$ \\
\hline$U+$ & $\{-\pi,-\pi / 2,0\}$ & $\{-1,0\}$ & $(-\pi, 0)$ & $(-\pi / 2,-1)$ & $(0,0)$ \\
\hline
\end{tabular}

In equation (4), the arbitrary constants $A$ and $B$ are assumed to be positive and non zero for this case. The direction of $\partial \mathrm{X}_{1}$ is changing with the qualitative magnitude of $X_{2}$ while the $X_{2} \in\{[-],[0],[+]\}$. The sinusoidal function has been defined in TABLE 3 , as $U$ (convex upward) or U+ (concave downward) constraints [2] in different operating regions and the two constants are qualitative variables. The exact difference of $[\mathrm{A}]$ and $[B]$ is unknown ([A] minus $[B]$ can be negative, zero and positive). Thus, there are three possible phase portraits for this set of confluence. The Fig. 6 shows the case while the difference of $[A]$ and $[B]$ is negative. Then, there are two possible points such that $\partial \mathrm{X}_{2}$ is zero and they are located at $X_{1} \in[0, \pi]$. If the operand sign is plus sign instead of minus sign in (4) and the difference of [A] and $[\mathrm{B}]$ is also negative, there are two zero points for $\partial \mathrm{X}_{2}$ and located at $X_{1} \in[-\pi, 0]$. The two points have been labeled as $\left(c_{1}, 0\right)$ and $\left(c_{2}, 0\right)$. 


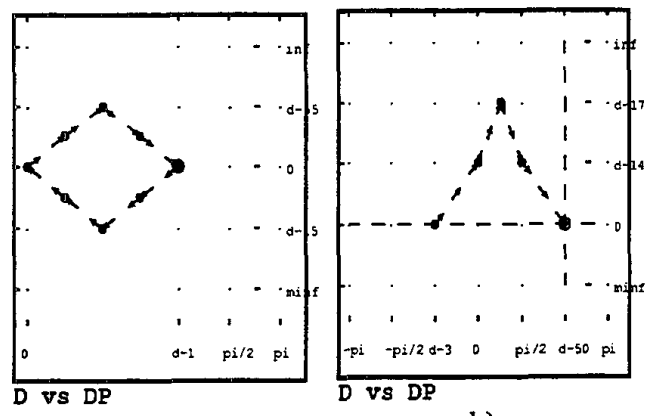

a)

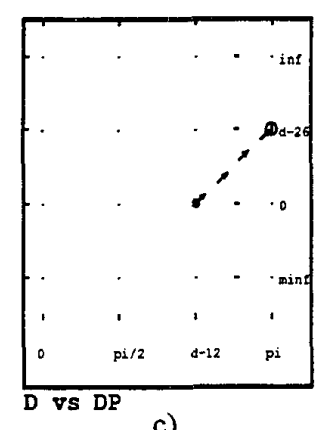

c)

Fig. 7: Two QSIM qualitative phase portraits

The Fig. 5 shows the partitioned phase plane regions and labeled them with the state numbers. The two corresponding isoclines are two vertical lines and partition the four regions into eight sub-regions. The number of states is $21(7 \times 3)$ states now. The possible trajectories can be illustrated from Fig. 6 according to those arrows. The state 1.5 is a diverge region, while trajectories, pass through this region, the model may be possible to diverge. The state 1.3 and 3.5 are converge regions, such that those trajectories pass through these two regions converge to the quiescent state $2.4\left(c_{2}, 0\right)$. There are a lot of possible trajectories can be derived from the qualitative phase portrait. The QSIM simulations, therefore, are helpful to illustrate the behaviors mutually.

\section{B. QSIM Simulations with Phase Portrait}

A model of simplified swing equation has been built in QSIM algorithm [2]. For this simulation, the interest operating regions of the system have been set to the range of rotor angle $\delta$ in $[-\pi, 0]$ and $[0, \pi]$, so there are transitions from one operating region into another region. The sinusoidal function would be described in each region by $\mathrm{U}+$ or $\mathrm{U}$ - constraints as in TABLE 3 [2]. The two fixed points, from the previous analysis, $c_{1}$ and $c_{2}$, haven't been provided into the corresponding values or landmarks. The simulation, however, predicts the possible fixed points. The non-intersection constraint
[11], [12] have been applied in the relationships of $\left(\delta, \delta^{\prime}\right)$ and $\left(\delta^{\prime}, \delta^{\prime \prime}\right)$ to reduce the spurious behaviors.

Before the simulation, initial states of the system have been set as $\delta$ is in $[-\pi, 0]$ and $[0, \pi]$ with the direction is unknown and the $\delta^{\prime}$ is 0 with direction is unknown too. There are 10 initial states found. Then, the QSIM finds all the possible initial states and propagates the following states. Three of the possible behaviors are shown in Fig. 7. It detects the cyclic behaviors in Fig. 7a, so there is a fixed point, which is located at the region of $\delta$ between 0 and $\pi / 2$, inside this limit cycle. Fig. $7 \mathrm{~b}$ shows a trajectory reached a quiescent state, which is located at the region of $\delta$ between $\pi / 2$ and $\pi$.

TABLE 4: STATE TRANSITIONS WITH FIG. 7

\begin{tabular}{|l|l|}
\hline Fig. 7a & $\begin{array}{l}2.1 \rightarrow 1.1 \rightarrow 1.2 \rightarrow 1.3 \rightarrow 2.3 \\
\rightarrow 3.3 \rightarrow 3.2 \rightarrow 3.1 \rightarrow 2.1\end{array}$ \\
\hline Fig. 7b & $2.1 \rightarrow 1.1 \rightarrow 1.2 \rightarrow 1.3 \rightarrow 2.4$ \\
\hline Fig. 7c & $2.5 \rightarrow 1.5$ \\
\hline
\end{tabular}

Those trajectories (Fig. 7a) are oscillating when they are near the $c_{1}$ and some of them rest on the $c_{2}$ but some of them leave from $c_{2}$. The three cases in Fig. 7 could be matched to the state transitions. For the Fig. 7a, the state transition is a complete cycle. The complete state transitions have been shown in the TABLE 4.

\section{CONCLUSION}

Qualitative analysis of the phase space does not need as many as the calculations compared with the numerical simulations. The characteristics of the system can be found faster and easier. The distinct behaviors have been shown after the qualitative analysis successfully, even through the exact numerical values haven't been shown (we also haven't provided the numerical values of the state-space models). The descriptions of qualitative analysis are clearer than the numerical analysis.

The phase space analysis [5], [7] and the qualitative simulation [2] can help engineers to predict and analyze the possible behaviors of a model, applied in the qualitative simulation is able to reduce the spurious behaviors. Using the qualitative phase plane analysis before QSIM, it would be helpful to be understood those behaviors. There, however, are some spurious behaviors in the outcome of the simulations.

The possible behaviors of the simplified swing equation have been described in qualitative methods successfully. In future, other electrical dynamical systems can be described by qualitative description even in time domain or phase portrait. 


\section{REFERENCE}

[1] B. M. Weedy, Electric Power Systems, John Wiley \& Sons, 1990

[2] Benjamin Kuipers, Qualitative Reasoning - Modeling and Simulation with Incomplete Knowledge, The MIT Press, 1994.

[3] D. P. Atherton, Nonlinear Control Engineering, Van nostrand Reinhold, 1982.

[4] De Kleer, J. and Brown, J. S., A Qualitative physics based on confluences, Arificial Intelligence, 24, 1984, p.7-83.

[5] Elisha P. Sacks, Automatic analysis of one-parameter planar ordinary differential equations by intelligent numeric simulation, Artificial Intelligence, Elsevier, Vol. 48, 1991, p.27-56.

[6] Hannes Werthner, Qualitative Reasoning: Modeling and the Generation of Behavior, Springer-Verlag Wien New York, 1994
[7] Jane Frances Macfarlane, Qualitative and Symbolic Analysis of Dynamic Physical Systems, Ph.D. Thesis, The university of Minnesota, UMI, 1989.

[8] P. A. Cook, Nonlinear dynamical systems, Second Edition, Prentice Hall, 1994

[9] Toyoaki Nishida, Grammatical description of behaviors of ordinary differential equations in two-dimensional phase space, Artificial Intelligence, Vol. 91, 1997, pp.3-32.

[10] William E. Boyce, Richard C. DiPrima, Elementary differential equations and boundary value problems, John Wiley \& Sons, Inc. New York, 1992

[11] Wood W. Lee and Benjamin J Kuipers, Non-Intersection of Trajectories in Qualitative Phase Space: A global Constraint for Qualitative Simulation, Proceedings of AAAI-88, p.286-290.

[12] Wood W. Lee and Benjamin J. Kuipers, A Qualitative Method to Construct Phase Portraits, Proceedings of AAA1-93, p.614-619 